\title{
Estudios para disminuir el síndrome metabólico en niños con obesidad: revisión sistemática
}

\author{
Studies to decrease metabolic syndrome in children with obesity: systematic review \\ Karina Straffon ${ }^{a}$ y Miroslava Porta-Lezama ${ }^{b}$
}

\begin{abstract}
:
Obesity is a public health problem characterized by being a chronic disease, caused by various factors ranging from genetic, metabolic or sociocultural problems.

Objective: To analyse studies or programs for the reduction of metabolic syndrome in children with obesity with favourable results. Method: A systematic online review was carried out based on articles published in PubMed using keywords such as "metabolic syndrome studies in children with obesity", "metabolic syndrome in children with obesity", "metabolic syndrome studies in children". Results: Initially, 37 studies or programs were found to decrease metabolic syndrome in children with obesity. When reviewing the programs, it was found that only 10 had favourable results in the participants.

Conclusion: In our country, being a place with high rates of obesity, both in children and adults, in addition to many chronic diseases, it is important to carry out more studies to find out more about this situation.
\end{abstract}

Keywords:

Metabolic syndrome, programs, reduction, children, obesity.

\section{Resumen:}

La obesidad es un problema de salud pública que se caracteriza por ser enfermedad crónica, causada por diversos factores que van desde problemas genéticos, metabólicos o socioculturales.

Objetivo: Analizar estudios o programas para la disminución de síndrome metabólico en niños con obesidad con resultados favorables.

Método: Se realizó una revisión sistemática en la red con base a artículos publicados en PubMed mediante el empleo de palabras claves como "estudios síndrome metabólico en niños con obesidad", "síndrome metabólico en niños con obesidad", "estudios síndrome metabólico en niños".

Resultados: Se encontraron en un inicio 37 estudios o programas para la disminución de síndrome metabólico en niños con obesidad, al revisar los programas se encontró que solo 10 tenían resultados favorables en los participantes.

Conclusión: En nuestro país siendo un lugar con altos índices de obesidad tanto infantil como en adultos, además de muchas enfermedades crónicas, es importante el desarrollo de más estudios para saber más de esta situación.

Palabras Clave:

Síndrome metabólico, programas, reducción, niños, obesidad

\section{INTRODUCCIÓN}

La obesidad es una enfermedad crónica, causada por diversos factores que van desde problemas genéticos, metabólicos o socioculturales, al respecto se ha referido que es un desequilibrio con tendencia negativa entre la ingesta alimentaria y el gasto calórico, ya que, se observa incremento en la primera

aAutor de Correspondencia, Universidad Autónoma del Estado de Hidalgo, https://orcid.org/0000-0002-5727-0111,

Email: karinastraffon94@gmail.com

bUniversidad Autónoma del Estado de Hidalgo, https://orcid.org/0000-0002-0952-6207,

Email: miroslava_porta8848@uaeh.edu.mx 
(sobreingesta rica en grasas y carbohidratos) y una disminución en la segunda (falta de actividad física o sedentarismo), lo que origina que el tejido adiposo se acumule y se eleve el peso corporal. ${ }^{1}$

En este sentido, las enfermedades no transmisibles se encuentran ampliamente relacionadas con la obesidad y representan un desafío importante, siendo la principal causa de muerte en todo el mundo. ${ }^{2}$ En los adultos se considera un conjunto de factores, conocidos como el "cuarteto mortal" (obesidad de la parte superior del cuerpo, intolerancia a la glucosa, hipertensión y colesterol) que aumentan el riesgo de sufrir enfermedad vascular cerebral, infartos o diabetes. ${ }^{3}$

Este conjunto de factores se puede encontrar con mayor frecuencia en la población pediátrica actualmente que hace 20 años, generado la necesidad de definir el síndrome metabólico infantil que se entiende para fines prácticos como una agrupación de factores de riesgo que afectan a la población infantil que incluyen principalmente obesidad central, hiperlipidemia de lipoproteínas de baja densidad (LDL), bajos niveles de lipoproteínas de alta densidad (HDL), que pueden presentar o no, algún grado de resistencia a la insulina e hipertensión arterial. ${ }^{2-3}$ Es importante señalar que no existe una definición única, lo que dificulta la estimación de la prevalencia del síndrome metabólico en esta población. ${ }^{4}$

Considerando que esta población rara vez presenta síntomas el dictamen de síndrome metabólico infantil más que un diagnostico se considera un indicador predictivo. Este diagnóstico no es universal debido al uso de criterios inconsistentes como por ejemplo los antecedentes familiares, el estado socioeconómico, la variación en los parámetros bioquímicos con la etnia. ${ }^{3}$

Los factores bioquímicos que intervienen en el desarrollo de estas enfermedades metabólicas se han estudiado con la esperanza de ayudar en su control o prevención. Los factores de crecimiento similares a la insulina (IGF) están involucrados en la regulación de las vías metabólicas y ejercen sus efectos sobre el sistema cardiovascular. ${ }^{5}$ En la infancia, el IGF-I se ha relacionado con marcadores de riesgo cardiovascular en adolescentes con obesidad, sin diabetes y se encontraron niveles bajos de IGF-I en niños chinos con obesidad con niveles bajos de colesterol de lipoproteína de alta densidad (HDLc). ${ }^{5}$

Las corrientes de investigación actuales sobre síndrome metabólico infantil presentan profundo interés en la prospectiva de diferentes factores de riesgo, basados en la teoría de que las circunstancias de la vida temprana pueden influir en el riesgo de enfermedad metabólica posterior, por ejemplo, desde la infancia empezamos a acumular lípidos en los vasos sanguíneos, fenómeno conocido como ateroesclerosis, que algún día se manifestará como enfermedades cardiovasculares. ${ }^{2}$

La obesidad es la acumulación de tejido adiposo y se asocia con la presencia de hipertensión, aumento de los niveles de triglicéridos, glucosa en sangre, el bajo nivel de colesterol HDL, liberación de ácidos grasos no esterificados y una multitud de adipocinas que inducen resistencia a la insulina, por lo tanto, se identifica como la base del síndrome metabólico. ${ }^{2,6}$

Dentro de los indicadores más utilizados para evaluar el exceso de peso tenemos los de composición corporal que incluye al índice de masa corporal (IMC), porcentaje de grasa corporal, circunferencia abdominal y grasa subcutánea. ${ }^{7}$ Por tanto, el objetivo de este estudio fue analizar estudios o programas para la disminución de síndrome metabólico en niños con obesidad con resultados favorables.

\section{MÉTODO}

Se realizó una revisión sistemática en la red con base en artículos publicados en PubMed mediante el empleo de palabras claves como "estudios síndrome metabólico en niños con obesidad", "síndrome metabólico en niños con obesidad", "estudios síndrome metabólico en niños”.

Los criterios de inclusión fueron los siguientes: artículos originales sobre estudios con población infantil ( $<18$ años), con objetivo definido, procedimiento detallado y resultados favorables. Mientras que los de exclusión fueron: revisiones sistemáticas, con participantes con enfermedades crónicas diagnosticadas dentro de la investigación y estudios o programas virtuales y/o a distancia.

\section{RESULTADOS}

Al realizar una búsqueda en PubMed se localizaron en una primera revisión 157 artículos sobre síndrome metabólico en niños con obesidad, pero al realizar una búsqueda más exhaustiva se encontraron que solo 37 artículos son sobre estudios para la disminución del síndrome metabólico en niños con obesidad que van desde 1999 hasta el 2019 (Figura 1).

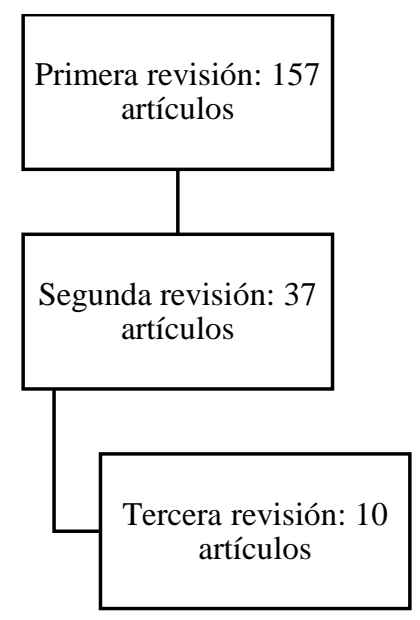

Figura 1. Proceso de la revisión sistemática

Finalmente, al revisar cada uno, buscando se cumplieran con todos los criterios de inclusión, se descartaron 27 artículos y se dejaron 10 dado que eran los únicos estudios sobre síndrome 
metabólico en niños con obesidad que tenían resultados favorables en sus participantes (Tabla 1).

Tabla 1. Comparación de estudios sobre síndrome metabólico en niños con obesidad.

\begin{tabular}{|c|c|c|c|c|c|}
\hline Autores y año & $\begin{array}{l}\text { Muestra y } \\
\text { duración }\end{array}$ & Objetivo & Intervención & Resultados & $\begin{array}{l}\text { Debilidad del } \\
\text { estudio }\end{array}$ \\
\hline $\begin{array}{l}\text { Ferguson, Gutin, } \\
\text { Le, Karp, } \\
\text { Litaker, } \\
\text { Humphries, } \\
\text { Okuyama, Riggs } \\
\text { y Owens (1999) }\end{array}$ & $\begin{array}{l}79 \text { niños y } \\
\text { niñas entre } 7 \\
\text { y } 11 \text { años de } \\
\text { edad con } \\
\text { obesidad } \\
\text { (pliegue } \\
\text { subcutáneo } \\
\text { tricipital } \\
\text { mayor al } \\
\text { percentl } 85 \\
\text { por sexo, } \\
\text { grupo étnico } \\
\text { y edad). } \\
\text { Duración: } 4 \\
\text { meses }\end{array}$ & 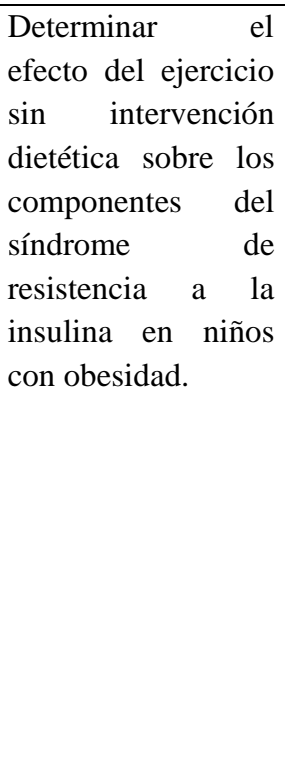 & \begin{tabular}{lr}
\multicolumn{2}{|c}{ Entrenamiento de } \\
ejercicio & 5 \\
días/semana & \\
durante & 40 \\
min/día &
\end{tabular} & 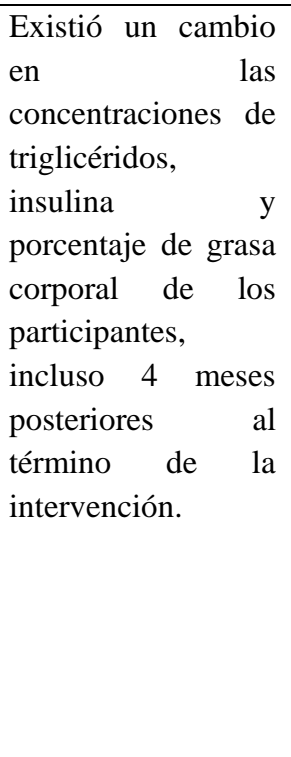 & $\begin{array}{lr}\text { No señala } & \text { si } \\
\text { durante el tiempo } \\
\text { posterior a la } \\
\text { intervención } & \text { fue } \\
\text { posible que los } \\
\text { participantes } \\
\text { mantuvieran } \\
\text { hábito de } \\
\text { práctica } \\
\text { ejercicio } \\
\text { cotidiano. }\end{array}$ \\
\hline $\begin{array}{l}\text { Kelly, } \\
\text { Wetzsteon, } \\
\text { Kaiser, } \\
\text { Steinberg, Bank } \\
\text { y Dengel (2004) }\end{array}$ & $\begin{array}{l}25 \text { hombres } \\
\text { y mujeres } \\
\text { niños y } \\
\text { adolescents } \\
\text { con exceso } \\
\text { de peso (por } \\
\text { arriba del } \\
\text { percentil } 85 \\
\text { del IMC para } \\
\text { la edad por } \\
\text { sexo). } \\
\text { Duración: } 8 \\
\text { semanas. }\end{array}$ & $\begin{array}{l}\text { Evaluar la } \\
\text { inflamación } \\
\text { subclínica y la } \\
\text { insulina en ayunas } \\
\text { como marcador de } \\
\text { resistencia a la } \\
\text { insulina y la función } \\
\text { endotelial para } \\
\text { determinar el efecto } \\
\text { del ejercicio } \\
\text { aeróbico sobre esas } \\
\text { variables. }\end{array}$ & 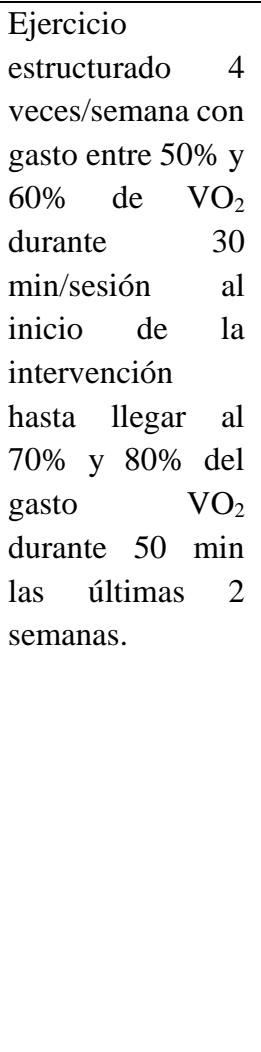 & $\begin{array}{l}\text { Los principales } \\
\text { cambios } \\
\text { encontraron en los } \\
\text { niveles de insulina y } \\
\text { en el colesterol de } \\
\text { lipoproteínas de alta } \\
\text { densidad (HDL), } \\
\text { pero no hubo } \\
\text { diferencias entre el } \\
\text { grupo intervenido y } \\
\text { el control respecto a } \\
\text { peso, porcentaje de } \\
\text { grasa corporal, } \\
\text { tensión arterial, } \\
\text { colesterol total, } \\
\text { colesterol LDL, } \\
\text { triglicéridos, } \\
\text { glucosa, insulina, } \\
\text { tolerancia a la } \\
\text { glucosa, entre otros. }\end{array}$ & $\begin{array}{lr}\begin{array}{l}\text { Aunque } \\
\text { observó que la }\end{array} \\
\text { insulina en ayuno } \\
\text { mejoró con el } \\
\text { ejercicio, no hubo } \\
\text { cambios en la } \\
\text { proteína } & \text { C } \\
\text { reactiva (como } \\
\text { marcador de la } \\
\text { inflamación) lo } \\
\text { cual puede } \\
\text { deberse a que el } \\
\text { peso corporal no } \\
\text { disminuyó, } \\
\text { consecuentemente } \\
\text { la grasa corporal } \\
\text { se mantuvo } \\
\text { similar, lo que } \\
\text { significa que los } \\
\text { adipocitos al no } \\
\text { cambiar, no hubo } \\
\text { modificación de la } \\
\text { respuesta } \\
\text { inflamatoria. }\end{array}$ \\
\hline $\begin{array}{l}\text { Young-Ran T, Ji- } \\
\text { Yeon A, Young- }\end{array}$ & $\begin{array}{l}32 \\
\text { participantes } \\
\text { con obesidad }\end{array}$ & $\begin{array}{lr}\text { Identificar } & \text { los } \\
\text { efectos de una } \\
\text { modificación de la }\end{array}$ & $\begin{array}{l}\text { Se hizo ejercicio } \\
\text { dos veces a la } \\
\text { semana por } 50\end{array}$ & $\begin{array}{l}\text { La circunferencia } \\
\text { de cintura, la } \\
\text { tensión arterial }\end{array}$ & $\begin{array}{lr}\text { No existe } & \text { grupo } \\
\text { control. } & \mathrm{La} \\
\text { información } & \mathrm{de}\end{array}$ \\
\hline
\end{tabular}


Hidalgo, Vol. 8, No. 16 (2020) 201-211

\begin{tabular}{|c|c|c|c|c|c|}
\hline $\begin{array}{l}\text { A K, Hae-Young } \\
\text { W }(2007)^{10}\end{array}$ & $\begin{array}{l}\text { (mayor del } \\
\text { percentil } 85 \\
\text { de las tablas } \\
\text { coreanas u } \\
\text { obesidad } \\
\text { relativa } \geq \\
10) \\
\\
\text { Duración: } 8 \\
\text { semanas. }\end{array}$ & $\begin{array}{l}\text { actividad física- } \\
\text { comportamiento } \\
\text { intervención } \\
\text { combinada } \\
\text { (intervención } \\
\text { PABM) sobre } \\
\text { metabolismo } \\
\text { factores de riesgo en } \\
\text { la escuela primaria } \\
\text { con sobrepeso y } \\
\text { obesidad niños. }\end{array}$ & $\begin{array}{l}\text { minutos (danza } \\
\text { hip-hop } \\
\text { gimnasia). } \\
\text { Instrucciones } \\
\text { para modificar } \\
\text { conductas (dieta y } \\
\text { ejercicio), por } 50 \\
\text { minutos una vez a } \\
\text { la semana. }\end{array}$ & $\begin{array}{l}\text { sistólica y diastólica } \\
\text { y el colesterol HDL, } \\
\text { se modificaron } \\
\text { significativamente. } \\
\text { No se modificaron } \\
\text { la glucosa ni los } \\
\text { triglicéridos }\end{array}$ & $\begin{array}{l}\text { pre y post } \\
\text { intervención } \\
\text { muestra aumentos } \\
\text { en las variables } \\
\text { significativas } \\
\text { (tensión arterial } \\
\text { sistólica } \\
\text { diastólica, } \\
\text { circunferencia de } \\
\text { la cintura y en } \\
\text { colesterol HDL) y } \\
\text { sin cambio en } \\
\text { glucosa } \\
\text { triglicéridos. Los } \\
\text { resultados } \\
\text { contradicen las } \\
\text { conclusiones de } \\
\text { otros autores } \\
\text { sobre el éxito de } \\
\text { las intervenciones }\end{array}$ \\
\hline $\begin{array}{l}\text { Kelishadi, } \\
\text { Hashemipour, } \\
\text { Mohammadifard, } \\
\text { Alikhassy y } \\
\text { Adeli }(2008)^{11}\end{array}$ & $\begin{array}{l}100 \text { niños y } \\
\text { niñas entre } 7 \\
\text { y } 9 \text { años de } \\
\text { edad con } \\
\text { obesidad } \\
\text { (IMC mayor } \\
\text { o igual al } \\
\text { percentil } 95 \\
\text { por sexo y } \\
\text { edad). }\end{array}$ & $\begin{array}{l}\text { Comparar a corto y } \\
\text { largo plazo cambios } \\
\text { en la concentración } \\
\text { de grelina después } \\
\text { de incrementar el } \\
\text { gasto energético vs. } \\
\text { los cambios por } \\
\text { disminuir la ingesta } \\
\text { energética r en } \\
\text { prepúberes r con } \\
\text { obesidad y así } \\
\text { determinar cuáles } \\
\text { son los factores } \\
\text { asociados con los } \\
\text { cambios en los } \\
\text { niveles de grelina } \\
\text { durante } \\
\text { disminución } \\
\text { soprepeso y del } \\
\text { asociación con el } \\
\text { síndrome } \\
\text { metabólico, } \\
\text { resistencia a la } \\
\text { insulina, medicines } \\
\text { antropométricas y } \\
\text { niveles de leptina. }\end{array}$ & $\begin{array}{l}\text { Se asignaron } 2 \\
\text { grupos, en uno se } \\
\text { implantó } \\
\text { entrenamiento } \\
\text { físico durante } 6 \\
\text { meses con } \\
\text { sesiones de } 40 \\
\text { min de actividad } \\
\text { aeróbica } \\
\text { días/semana, con } \\
\text { 15 min previos de } \\
\text { sesiones } \\
\text { sensibilización } \\
\text { para ser } \\
\text { mantenerse } \\
\text { activos. } \\
\text { El otro grupo } \\
\text { recibió } \\
\text { intervención } \\
\text { dietética } \\
\text { régimen en } \\
\text { restricción, de } \\
\text { conducido por un } \\
\text { profesional en el } \\
\text { área basado en la } \\
\text { energía requerida } \\
\text { para la talla. }\end{array}$ & $\begin{array}{l}\text { La reducción del } \\
\text { sobrepeso en } \\
\text { prepúberes a través } \\
\text { de establecer } \\
\text { durante } 6 \text { meses un } \\
\text { balance energético } \\
\text { negativo, ya sea por } \\
\text { actividad física o } \\
\text { por dieta, se reflejó } \\
\text { en el incremento } \\
\text { progresivo de las } \\
\text { concentraciones de } \\
\text { grelina en suero, } \\
\text { incluso un año } \\
\text { después del tiempo } \\
\text { cero. la } \\
\text { El incremento de la } \\
\text { grelina puede ser } \\
\text { gracias a la } \\
\text { reducción de peso y } \\
\text { consecuentemente } \\
\text { disminución del } \\
\text { tejido adiposo y no } \\
\text { necesariamente por } \\
\text { la restricción } \\
\text { dietética. } \\
\text { La grelina basal } \\
\text { tuvo una fuerte } \\
\text { correlación negativa } \\
\text { con las medidas de } \\
\text { obesidad central. } \\
\text { Con } 37 \% \text { más de }\end{array}$ & $\begin{array}{l}\text { Ante la falta de } \\
\text { estándares } \\
\text { universales para } \\
\text { definir } \\
\text { síndrome } \\
\text { metabólico en } \\
\text { niños, emplearon } \\
\text { los criterios de las } \\
\text { Guías de } \\
\text { tratamiento para } \\
\text { disminuir los } \\
\text { lípidos y el riesgo } \\
\text { de enfermedad } \\
\text { coronaria } \\
\text { diseñadas por el } \\
\text { National } \\
\text { Cholesterol } \\
\text { Education } \\
\text { Program Adult } \\
\text { Treatment Panel } \\
\text { III (ATP-III). } \\
\text { La modificación } \\
\text { de los estilos de } \\
\text { vida implica tanto } \\
\text { cambios en la } \\
\text { alimentación } \\
\text { como incremento } \\
\text { de la actividad } \\
\text { física, pero en este } \\
\text { estudio solamente } \\
\text { se enfocó a uno u } \\
\text { otro. }\end{array}$ \\
\hline
\end{tabular}


Hidalgo, Vol. 8, No. 16 (2020) 201-211

\begin{tabular}{|c|c|c|c|c|c|}
\hline & & & & $\begin{array}{l}\text { probabilidades } \\
\text { síndrome } \\
\text { metabólico en los } \\
\text { participantes con } \\
\text { niveles bajos de } \\
\text { grelina. }\end{array}$ & \\
\hline $\begin{array}{l}\text { Reinehr, Kleber } \\
\text { y Toschke } \\
(2009)^{12}\end{array}$ & 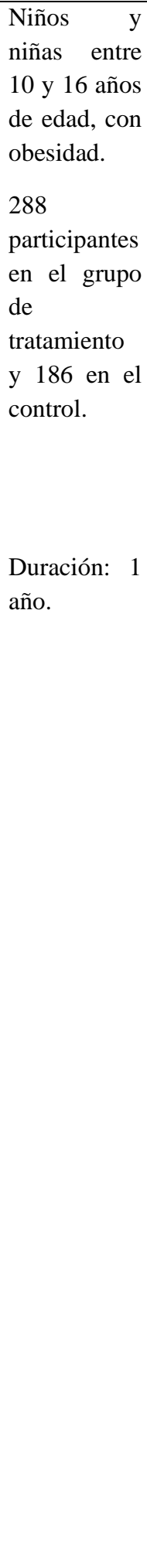 & $\begin{array}{l}\text { Evaluar un cambio } \\
\text { en la prevalencia del } \\
\text { síndrome } \\
\text { metabólico basado } \\
\text { en diferentes } \\
\text { definiciones, } \\
\text { incluidas } \\
\text { pruebas las } \\
\text { tolerancia a la } \\
\text { glucosa en niños } \\
\text { con obesidad bajo } \\
\text { intervención de } \\
\text { estilo de vida. }\end{array}$ & 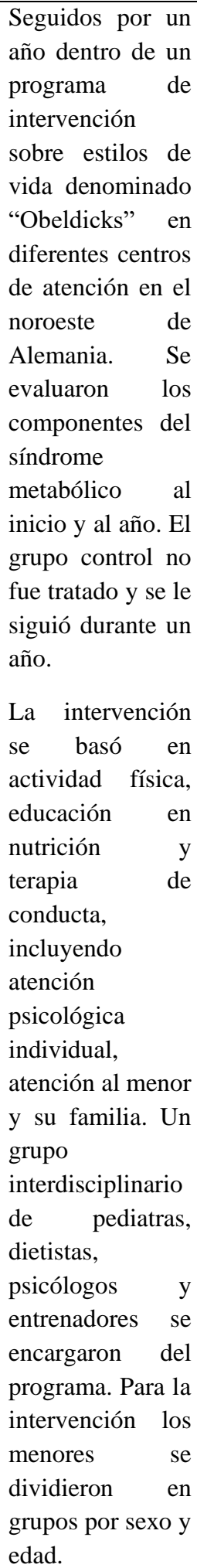 & 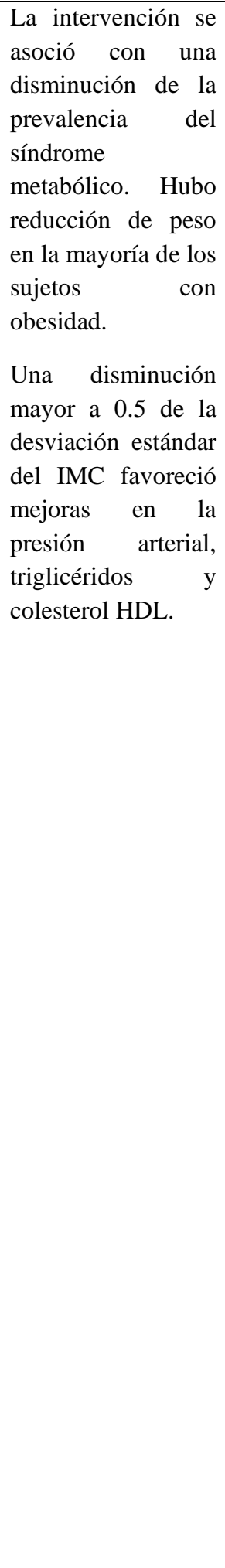 & \begin{tabular}{lr} 
El grupo control \\
se eligió & de \\
familias & de \\
menores & que \\
\multicolumn{2}{c}{ vivían lejos de los } \\
centros & de \\
tratamiento o & que \\
carecían & de \\
medios & de \\
transporte. & No \\
tiene & un \\
seguimiento & \\
posterior & para \\
evaluar & la \\
permanencia & de \\
los cambios. &
\end{tabular} \\
\hline
\end{tabular}


Hidalgo, Vol. 8, No. 16 (2020) 201-211

\begin{tabular}{|c|c|c|c|c|c|}
\hline & & & $\begin{array}{ll}\text { Los } & \text { padres } \\
\text { también fueron } & \text { fuecitados }\end{array}$ & & \\
\hline $\begin{array}{l}\text { Wickham, Stern, } \\
\text { Evans, Bryan, } \\
\text { Moskowitz, } \\
\text { Clore y Laver } \\
(2009)^{13}\end{array}$ & $\begin{array}{l}165 \\
\text { adolescentes } \\
\text { entre } 11 \text { y } 18 \\
\text { años de edad } \\
\text { con obesidad } \\
\text { (IMC mayor } \\
\text { o igual al } \\
\text { percentil } 95 \\
\text { por sexo y } \\
\text { edad). } \\
\begin{array}{l}\text { Duración: } 6 \\
\text { meses }\end{array}\end{array}$ & $\begin{array}{l}\text { Determinar la } \\
\text { prevalencia } \\
\text { síndrome } \\
\text { metabólico al inicio } \\
\text { del estudio y } \\
\text { después de } 6 \text { meses } \\
\text { de modificación del } \\
\text { estilo de vida entre } \\
\text { adolescentes con } \\
\text { obesidad, como } \\
\text { parte de un } \\
\text { programa } \\
\text { multidisciplinario } \\
\text { de control de peso. }\end{array}$ & $\begin{array}{lr}\text { Los } r & \text { sujetos } \\
\text { fueron invitados a } \\
\text { realizar al menos } \\
1 \quad \text { hora, } \\
\text { días/semana en } \\
\text { sesiones entre } 60 \\
\text { y } 80 \text { min de } \\
\text { actividad } \\
\text { aeróbica y de } \\
\text { resistencia, } \\
\text { acuerdo con el } \\
\text { monitoreo de la } \\
\text { frecuencia } \\
\text { cardiaca de cada } \\
\text { uno. }\end{array}$ & $\begin{array}{l}\text { Después de } 6 \text { meses } \\
\text { de modificación del } \\
\text { estilo de vida, el } \\
\text { IMC, porcentaje de } \\
\text { grasa, colesterol } \\
\text { total y lipoproteína } \\
\text { de baja densidad el } \\
\text { colesterol (LDL-C) } \\
\text { disminuyeron } \\
\text { significativamente. } \\
\text { La resistencia a la } \\
\text { insulina no tuvo } \\
\text { cambios. } \\
\text { El número de } \\
\text { componentes (entre } \\
3 \text { y } 4 \text { ) que definen el } \\
\text { síndrome } \\
\text { metabólico no } \\
\text { cambió al final del } \\
\text { estudio. }\end{array}$ & $\begin{array}{lr}\text { Los cambios en } \\
\text { los estilos de vida } \\
\text { aunque logran } \\
\text { modificar varios } \\
\text { componentes } & \text { del } \\
\text { síndrome } & \\
\text { metabólico romo } \\
\text { el IMC, } \\
\text { porcentaje }\end{array}$ \\
\hline $\begin{array}{l}\text { Hashemipour, } \\
\text { Kelishadi, } \\
\text { Shapouri, } \\
\text { Sarrafzadegan, } \\
\text { Amini, Tavakoli, } \\
\text { Movahedian- } \\
\text { Attar, } \\
\text { Mirmoghtadaee } \\
\text { y Poursafa } \\
(2009)^{14}\end{array}$ & $\begin{array}{lr}60 \text { menores } \\
\text { entre } 6 \text { y } 10 \\
\text { años de } \\
\text { edad, con } \\
\text { obesidad. }\end{array}$ & $\begin{array}{l}\text { Evaluar el efecto de } \\
\text { la suplementación } \\
\text { con zinc sobre la } \\
\text { resistencia a la } \\
\text { insulina y los } \\
\text { componentes del } \\
\text { síndrome } \\
\text { metabólico en niños } \\
\text { con obesidad } \\
\text { prepúberes. }\end{array}$ & 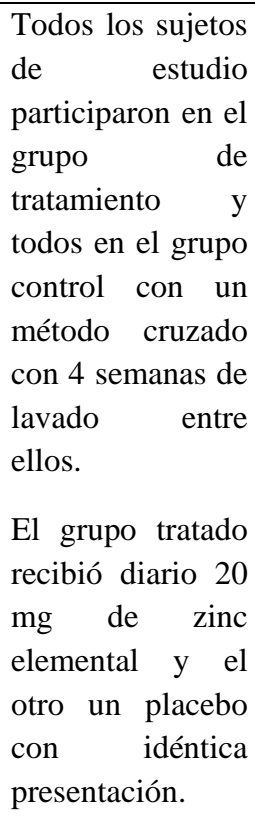 & $\begin{array}{l}\text { Después de recibir } \\
\text { zinc, la glucosa } \\
\text { plasmática media en } \\
\text { ayunas, la insulina, } \\
\text { el HOMA-IR y el } \\
\text { IMC disminuyeron } \\
\text { significativamente. } \\
\text { La circunferencia } \\
\text { de la cintura, el } \\
\text { colesterol LDL y los } \\
\text { triglicéridos no } \\
\text { cambiaron. }\end{array}$ & $\begin{array}{l}\text { Un corto período } \\
\text { de intervención. } \\
\text { La dosis de zinc } \\
\text { estimada con base } \\
\text { en la experiencia } \\
\text { en adultos, un } \\
\text { período corto de } \\
\text { lavado que se } \\
\text { tradujo } \\
\text { resultados menos } \\
\text { efectivos } \\
\text { comparar } \\
\text { grupos cuando los } \\
\text { tratados pasaron a } \\
\text { ser controles. }\end{array}$ \\
\hline $\begin{array}{l}\text { Pedrosa, } \\
\text { Oliveira, } \\
\text { Albuquerque, } \\
\text { Simões-Pereira, } \\
\text { Vaz-de-Almeida } \\
\text { y Correia } \\
(2010)^{15}\end{array}$ & $\begin{array}{l}83 \text { niños } \mathrm{y} \\
\text { niñas entre } 7 \\
\text { y } 9 \text { años de } \\
\text { edad con } \\
\text { sobrepeso u } \\
\text { obesidad } \\
\text { (IMC mayor } \\
\text { o igual al }\end{array}$ & $\begin{array}{l}\text { Evaluar los cambios } \\
\text { en el grado de } \\
\text { sobrepeso/obesidad, } \\
\text { la prevalencia de } \\
\text { síndrome } \\
\text { metabólico y sus } \\
\text { componentes en } \\
\text { niños escolares con } \\
\text { sobrepeso }\end{array}$ & $\begin{array}{l}\text { Programa de } \\
\text { intervención de } \\
\text { estilo de vida: } \\
\text { asesoramiento en } \\
\text { nutrición con una } \\
\text { recomendación } \\
\text { dietaria y de } \\
\text { ejercicio. Los } \\
\text { grupos }\end{array}$ & $\begin{array}{l}\text { Ambos tipos de } \\
\text { intervención } \\
\text { tuvieron resultados } \\
\text { similares por } \\
\text { cambios en los } \\
\text { estilos de vida, } \\
\text { condujeron a la } \\
\text { mejora en el IMC, } \\
\text { índice }\end{array}$ & $\begin{array}{l}\text { La disminución } \\
\text { del tamaño de la } \\
\text { muestra fue } \\
\text { paulatina a lo } \\
\text { largo del estudio, } \\
\text { de tal manera que } \\
\text { de } 83 \text { sujetos, lo } \\
\text { terminaron } 61 \text {, es }\end{array}$ \\
\hline
\end{tabular}


Hidalgo, Vol. 8, No. 16 (2020) 201-211

\begin{tabular}{|c|c|c|c|c|c|}
\hline & $\begin{array}{l}\text { percentil } \\
85) . \\
\text { Duración: } 1 \\
\text { año. }\end{array}$ & $\begin{array}{ll}\text { obesidad } & \\
\text { participantes en } & \text { un } \\
\text { programa } & \text { de } \\
\text { intervención. } & \end{array}$ & 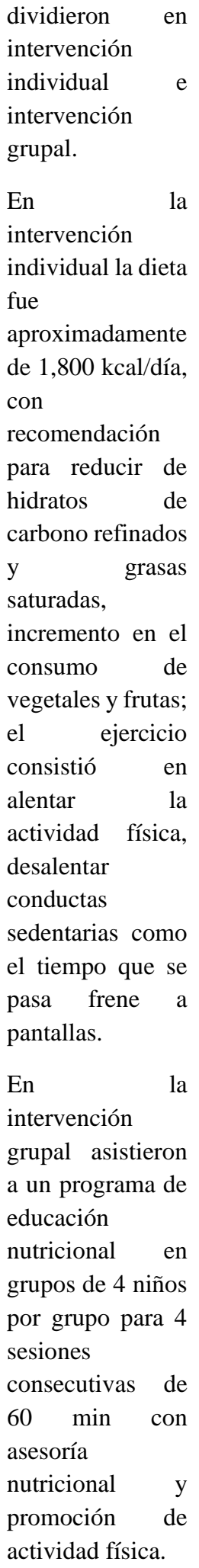 & $\begin{array}{l}\text { circunferencia de } \\
\text { cintura/altura, } \\
\text { colesterol HDL, } \\
\text { triglicéridos, } \\
\text { apolipoproteína A-I } \\
\text { y B. } \\
\text { La prevalencia de } \\
\text { síndrome } \\
\text { metabólico } \\
\text { disminuyó a 14.8\% } \\
\text { de 16.4\% en niños } \\
\text { con obesidad. } \\
\text { El número de } \\
\text { componentes del } \\
\text { síndrome } \\
\text { metabólico fue más } \\
\text { alto en niños con } \\
\text { IMC mayor. } \\
\text { Los niños con } \\
\text { sobrepeso/obesidad, } \\
\text { comparados con los } \\
\text { de peso normal } \\
\text { presentaron valores } \\
\text { más altos de tensión } \\
\text { arterial, colesterol } \\
\text { total, triglicéridos, } \\
\text { apolipoproteína B y } \\
\text { proteína C reactiva. }\end{array}$ & $\begin{array}{l}\text { decir, se perdió el } \\
26.5 \% \text {. }\end{array}$ \\
\hline $\begin{array}{l}\text { Velázquez- } \\
\text { López, Santiago- } \\
\text { Díaz, Nava- } \\
\text { Hernández, } \\
\text { Muñoz-Torres, } \\
\text { Medina-Bravo, }\end{array}$ & $\begin{array}{l}44 \text { niños y } \\
\text { adolescentes } \\
\text { de uno u otro } \\
\text { sexo con } \\
\text { edad }\end{array}$ & $\begin{array}{lr}\text { Evaluar la } & \text { eficacia } \\
\text { de la } & \text { terapia } \\
\text { utilizando } & \text { dieta } \\
\text { mediterránea } & \text { para } \\
\text { disminuir } & \\
\text { indicadores } & \text { de }\end{array}$ & $\begin{array}{l}16 \text { semanas de } \\
\text { dieta tipo } \\
\text { mediterránea en } \\
\text { el grupo de } \\
\text { tratamiento vs } \\
\text { dietas estándar. }\end{array}$ & $\begin{array}{l}\text { El grupo de dieta } \\
\text { mediterránea, en } \\
\text { comparación con el } \\
\text { de dieta estándar } \\
\text { tuvo una } \\
\text { disminución }\end{array}$ & $\begin{array}{l}\text { La corta duración } \\
\text { del estudio, el no } \\
\text { evaluar el efecto } \\
\text { post tratamiento. } \\
\text { El no tener un }\end{array}$ \\
\hline
\end{tabular}


Hidalgo, Vol. 8, No. 16 (2020) 201-211

\begin{tabular}{|c|c|c|c|c|c|}
\hline $\begin{array}{l}\text { Torres-Tamayo } \\
(2014)^{16}\end{array}$ & $\begin{array}{l}\text { promedio de } \\
11 \text { años. } \\
\text { Con IMC } \\
\text { mayor del } \\
\text { percentil } 90 . \\
\text { Duración: } 16 \\
\text { semanas. }\end{array}$ & $\begin{array}{l}\text { síndrome } \\
\text { metabólico en niños } \\
\text { y adolescentes con } \\
\text { obesidad. }\end{array}$ & $\begin{array}{l}\text { Se determinaron } \\
\text { indicadores } \\
\text { metabólicos y } \\
\text { antropométricos, } \\
\text { se hizo } \\
\text { evaluación } \\
\text { dietética en } \\
\text { estado basal y a } \\
\text { las } 16 \text { semanas. } \\
\text { Cada tres } \\
\text { semanas se hizo } \\
\text { evaluación } \\
\text { dietética, } \\
\text { antropométrica y } \\
\text { se resolvieron } \\
\text { dudas sobre las } \\
\text { indicaciones. } \\
\text { Ambos grupos } \\
\text { recibieron } \\
\text { orientación sobre } \\
\text { actividad física }\end{array}$ & $\begin{array}{l}\text { significativa en el } \\
\text { IMC, masa magra, } \\
\text { masa grasa, } \\
\text { glucosa, TC, TG, } \\
\text { HDL-C y LDL-C. } \\
\text { También disminuyó } \\
\text { los niveles de } \\
\text { glucosa y la } \\
\text { frecuencia } \\
\text { glucosa mayor a } \\
100 \mathrm{mg} / \mathrm{dL} \text {. }\end{array}$ & $\begin{array}{l}\text { indicador de } \\
\text { actividad física. }\end{array}$ \\
\hline $\begin{array}{l}\text { Niklowitz, } \\
\text { Rothermel, Lass, } \\
\text { Barth y Reinehr } \\
(2018)^{17}\end{array}$ & $\begin{array}{l}111 \\
\text { menores: } 88 \\
\text { participantes } \\
\text { del } \\
\text { programa } \\
\text { "Obeldicks"; } \\
23 \text { menores } \\
\text { con peso } \\
\text { normal y } \\
\text { sanos. } \\
\\
\text { Duración: } 1 \\
\text { año. }\end{array}$ & 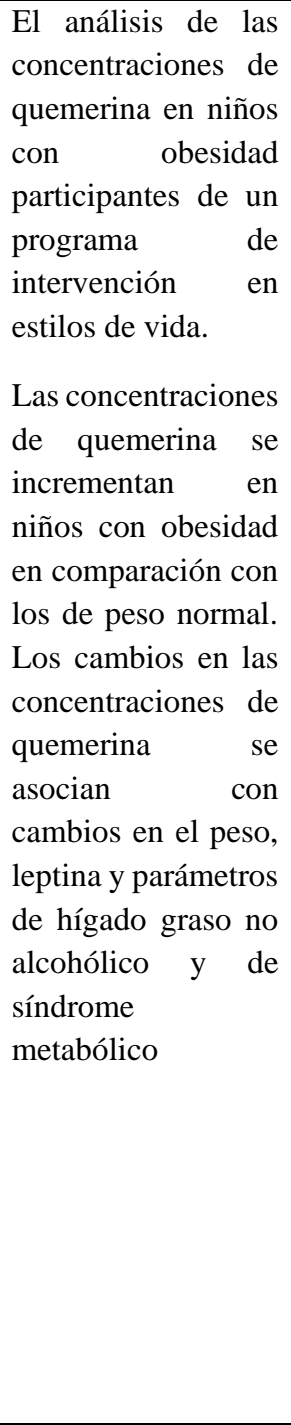 & 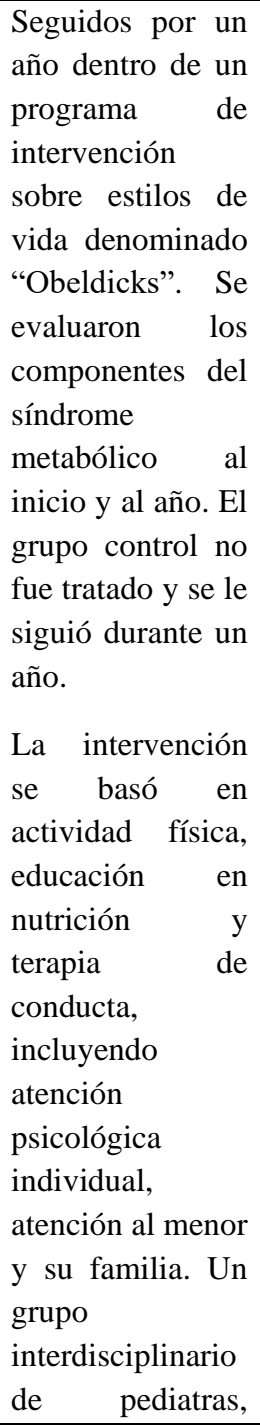 & 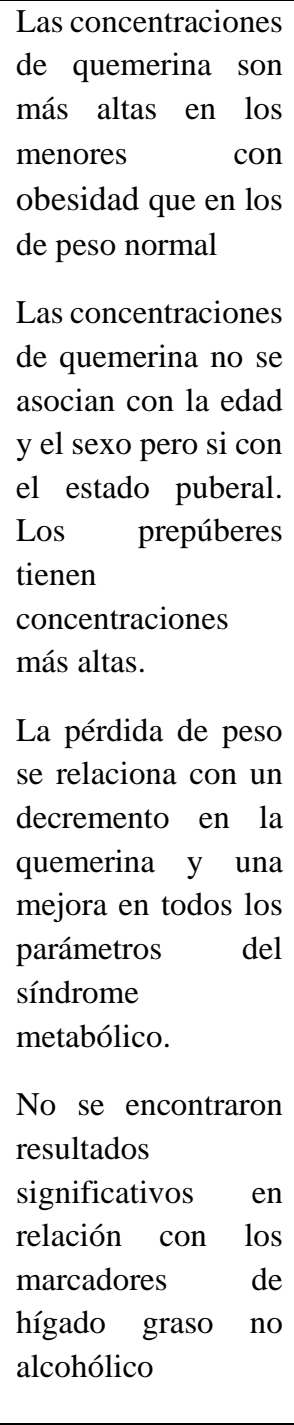 & 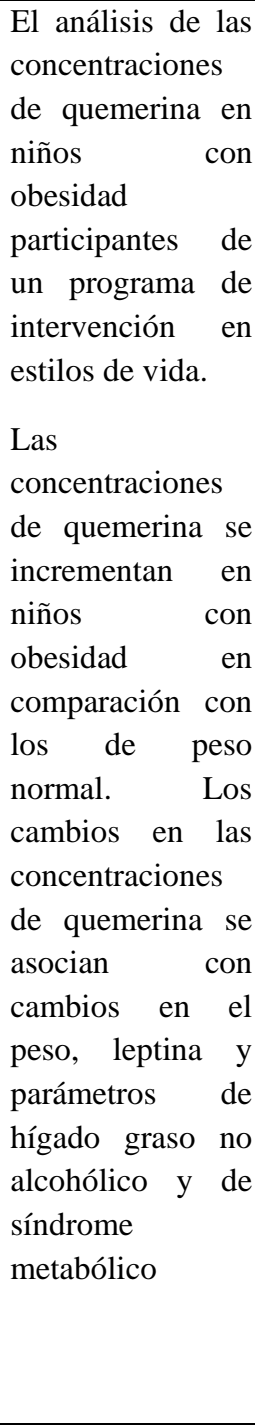 \\
\hline
\end{tabular}




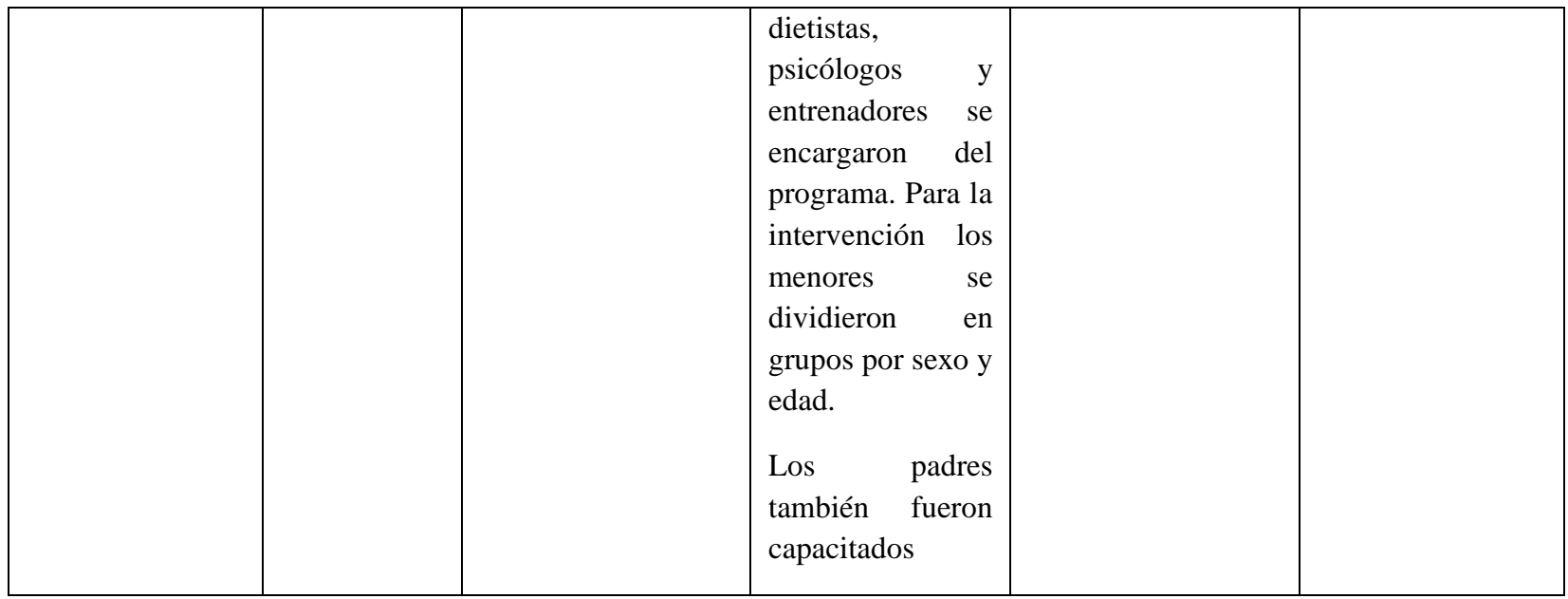

El síndrome metabólico es una enfermedad que no solo se ve presente en población adulta, sino que ahora las alteraciones en diversos sistemas del organismo se presentan en edades más tempranas. ${ }^{2}$ Comúnmente, más no siempre, se ve acompañado por sobrepeso y obesidad, demostrando que tener un exceso de peso se convierte en un factor de riesgo para esta enfermedad. ${ }^{1}$

En el presente reporte se buscaron estudios de intervención exitosa para población en edad pediátrica con obesidad y presencia o indicadores de síndrome metabólico, encontrando evidencia de la presencia de esta enfermedad a partir de los cinco años de edad. ${ }^{9}$

En los estudios encontrados, las intervenciones que realizaron los investigadores se enfocan principalmente al cambio de hábitos alimentarios y de actividad física, dado que indicaban que el estilo de vida que llevaban los participantes no era el adecuado y se demostró con grupos control que, de no modificarlos, a largo plazo podrían desencadenar en comorbilidades que reducen la calidad de vida o generan una muerte prematura. ${ }^{8,9,10,11,13}$

Los estudios más relevantes arrojan resultados similares a lo conocido para población adulta, pero en este reporte se presenta la evidencia científica para población en edad pediátrica. Se sostienen las recomendaciones que se han establecido previamente respecto a la actividad física regular donde el efecto benéfico permanece incluso meses después de haberlo realizado, ${ }^{8}$ o el incremento de la capacidad aeróbica con tan solo 8 semanas de entrenamiento. ${ }^{9}$

Entre los factores que se benefician son desde luego los que conforman al síndrome metabólico como la obesidad, en algunos la abdominal específicamente, la tensión arterial, colesterol HDL, resistencia a la insulina, glucosa, y otros componentes relacionados como triglicéridos. ${ }^{8-17}$

Entre las aportaciones novedosas, aunque no directamente relacionadas con la definición del síndrome metabólico, está la evaluación del porcentaje de grasa corporal, $, 8,911,13,16$ índice circunferencia de cintura/altura, ${ }^{15} \mathrm{o}$ bien, las concentraciones de grelina, regulada indirectamente por cambios en los hábitos alimentarios y de actividad física, ${ }^{11}$ así como las concentraciones de quemerina en niños con obesidad, la cual al mejorar los estilos de vida relacionados con alimentación y actividad física provocando disminución del peso corporal, disminuye esta adipocitocina y todos los parámetros del síndrome metabólico. ${ }^{17}$

Otro aspecto novedoso fue la suplementación con zinc durante 8 semanas en menores con obesidad para disminuir la resistencia a la insulina, la glucosa en ayuno y el IMC que este caso, resultó exitoso. ${ }^{14}$ Es consistente también el efecto de la actividad física y mejora de los hábitos alimentarios en la disminución de la proteína $\mathrm{C}$ reactiva, ${ }^{15}$ como indicador de inflamación.

Pedrosa menciona además horas de sueño como un elemento importante que forma parte de los estilos de vida y el tiempo que se dedica viendo televisión. Los niños con sobrepeso/obesidad duermen menos, pasan más tiempo frente a una pantalla que quienes tienen peso normal y participan menos en actividades extracurriculares que demandan ejercicio. ${ }^{15}$

Finalmente, la participación de la familia resulta importante en las estrategias de intervención, así como la asesoría psicológica para los involucrados en el manejo de la obesidad y el síndrome metabólico en su conjunto. ${ }^{11,17}$

\section{DISCUSIÓN}

A diferencia de la población infantil, la obesidad en adultos es de las principales determinantes del síndrome metabólico, de hecho, forma parte de su definición en diversas propuestas de organismos internacionales como la del Grupo de Trabajo de la Federación Internacional de Diabetes en Epidemiología y Prevención, Asociación Americana del Corazón/Instituto Nacional del Corazón, los Pulmones y la Sangre (AHE/NHLBI, por sus siglas en inglés)-Guías del ATP III, Sociedad Internacional de Aterosclerosis, y la Asociación Internacional de Estudios de la Obesidad. ${ }^{18}$

La obesidad, en particular la abdominal que puede ser evaluada a través de la circunferencia de cintura, es un predictor de 
morbilidad y mortalidad, incluso en personas con IMC normal, lo cual se hace evidente de manera indirecta en la población infantil al responder de manera positiva a los cambios de los estilos de vida. ${ }^{19}$

Debido a que México por un lado sigue siendo un país con grandes disparidades en cuanto al acceso y uso de los servicios de salud, y por otro lado la evidente falta de preparación en los modelos de atención para el tratamiento exitoso de este tipo de problemáticas, contamos con amplias áreas de oportunidad para los programas de salud. ${ }^{20}$

Es importante señalar que el síndrome metabólico en población infantil no se puede medir con facilidad debido a la falta de estándares claros y valores universales para ese grupo de edad, situación que no se observa en adultos para quienes se cuenta con criterios definidos. ${ }^{4,5}$ Por lo anterior, actuar en poblaciones en edades tempranas sobre el conocimiento de nutrimentos y la composición de una dieta correcta, podría ayudar a sacar a la población de un contexto de consumismo alimentario. ${ }^{21}$

En la actualidad, la prevalencia del síndrome metabólico se ha incrementado en la población y como se hace evidente en los diversos estudios, evitarlo es posible con adecuaciones en los estilos de vida. No obstante, este síndrome en el que la obesidad es el principal detonador de las enfermedades que lo conforman, no es determinante; en un estudio en el que participaron adultos estadounidenses sin obesidad, el $3.5 \%$ de quienes padecen síndrome metabólico; mientras que en otra población estadounidense con obesidad, el $14.5 \%$ tenían este síndrome, es decir, cuatro veces más que aquellos que no tienen obesidad. Este análisis es más contundente en nuestro país, dado que mientras el $9.4 \%$ de la población sin obesidad tiene síndrome metabólico, el $44.2 \%$ de quienes padecen obesidad, también presentan el síndrome. ${ }^{4,8,9,10,11}$

La población de nuestro país presenta alta prevalencia de obesidad abdominal en adultos: el $65.4 \%$ de hombres y $87.7 \%$ de mujeres, y respecto a la dieta, de acuerdo con la propuesta de la Organización Mundial de la Salud, menos del 35\% de la población adulta consume la ingesta recomendada de verduras y frutas, en contraste con el alto consumo de bebidas no lácteas endulzadas ( $85.8 \%$ de adultos), ${ }^{22}$ lo que puede traducirse en un futuro en la carga de enfermedad para el sistema de salud mexicano, llegando incluso rebasar su capacidad. Esto es relevante si se considera que en población con menor edad (de 1 a 19 años) menos del 50\% consume cotidianamente verduras y frutas, aunado al alto consumo de bebidas no lácteas endulzadas (por arriba del $80 \%$ ) y de botanas, dulces y postres (entre el $54 \%$ y $65 \%$ ) y se suma también el exceso de peso corporal que va del $30 \%$ al $40 \%$ en este mismo grupo de edad, ${ }^{23}$ lo cual se dirige a una potencial sobrecarga en los servicios de salud, rebasando incluso la demanda que hoy tenemos.

\section{CONCLUSIÓN}

Las evidencias más contundentes en cuanto a las intervenciones exitosas para el tratamiento del síndrome metabólico en población infantil y adolescente no distan de lo publicado para población adulta. Los puntos focales de intervención son los cambios en la alimentación y el incremento de la actividad física, ambos constituyen los elementos más contundentes en los estilos de vida saludables y que se reflejan en el desarrollo de enfermedades crónicas. Para este grupo de edad también destaca la participación de la familia y asesoramiento psicológico para los niños y sus padres. Aunque la obesidad no siempre está presente en el síndrome metabólico en todos los grupos de edad, dado que es el principal detonador para el desarrollo de la lipoinflamación y en consecuencia de la resistencia a la insulina, diabetes, hipertensión, dislipidemias y enfermedad cardiovascular, es necesario seguir tomándolo como punto de partida para la prevención y tratamiento de cualquiera de estas enfermedades.

En nuestro país, siendo un lugar con altos índices de obesidad en todos los grupos de edad, además de la presencia de diversas enfermedades crónicas, o un conjunto de ellas como lo es el síndrome metabólico, es necesario el desarrollo de estudios que permitan tener mayor claridad sobre estrategias desde la perspectiva de la salud pública para la prevención, control y manejo de este tipo de enfermedades, enfocados en la formación de hábitos y estilos de vida estructurados en los que puedan estar involucrados la familia y no solamente un miembro de esta.

Los determinantes sociales de la enfermedad se hacen evidentes en enfermedades como esta, las estrategias que se han diseñado siguen en el afán de combatir una enfermedad que no es solamente biológica, sino social. Algunas de las estrategias como el etiquetado frontal de los alimentos, el incremento de los impuestos en algunos alimentos, la eliminación de dibujos y personajes animados para la promoción de alimentos y bebidas procesados, especialmente dirigidos a población en edades tempranas, son algunas de los intentos por combatir el ambiente obesigénico, no obstante, las estrategias básicas siguen siendo consistentes en cuanto a la alimentación correcta y la realización de actividad física que en edades escolares debería ser por lo menos de 60 minutos diarias, de acuerdo con lo señalado por la Organización Mundial de la Salud.

\section{CONFLICTO DE INTERESES}

Los autores declaramos que no tenemos conflicto de intereses.

\section{REFERENCIAS}

[1] Macías M AI, Gordillo S LG, Camacho R EJ. Hábitos alimentarios de niños en edad escolar y el papel de la educación para la salud. Rev. Chil. Nutr. 2012;39(3):40-43.

[2] Gustafsson J. Metabolic syndrome in children. Acta Paediatr. 2019;108(3):394-395. 
[3] Titmuss AT, Srinivasan S. Metabolic syndrome in children and adolescents: Old concepts in a young population. $\mathrm{J}$ Paediatr Child Health. 2016;52(10):928-934.

[4] Romero-Velarde E, Aguirre-Salas LM, Álvarez-Román YA, Vásquez-Garibay EM, Casillas-Toral E, Fonseca-Reyes S. Prevalencia de síndrome metabólico y factores asociados en niños y adolescentes con obesidad [Prevalence of metabolic syndrome and associated factors in children and adolescents with obesity]. Rev Med Inst Mex Seguro Soc. 2016;54(5):568-575.

[5] Inzaghi E, Baldini Ferroli B, Fintini D, Grossi A, Nobili V, Cianfarani S. Insulin-Like Growth Factors and Metabolic Syndrome in Obese Children. Horm Res Paediatr. 2017;87(6):400-404.

[6] Folić N, Folić M, Marković S, Andjelković M, Janković S. Risk factors for the development of metabolic syndrome in obese children and adolescents. Srp Arh Celok Lek. 2015;143(3-4):146-152.

[7] Gobato AO, Vasques AC, Zambon MP, Barros Filho Ade A, Hessel G. Metabolic syndrome and insulin resistance in obese adolescents. Rev Paul Pediatr. 2014;32(1):55-62.

[8] Ferguson MA, Gutin B, Le NA, et al. Effects of exercise training and its cessation on components of the insulin resistance syndrome in obese children. Int $\mathrm{J}$ Obes Relat Metab Disord. 1999;23(8):889-895.

[9] Kelly AS, Wetzsteon RJ, Kaiser DR, Steinberger J, Bank AJ, Dengel DR. Inflammation, insulin, and endothelial function in overweight children and adolescents: the role of exercise. J Pediatr. 2004;145(6):731-736.

[10] Young-Ran T, Ji-Yeon A, Young-A K, Hae-Young W. The Effects of a Physical Activity-Behavior Modification Combined Intervention(PABM-intervention) on Metabolic Risk Factors in Overweight and Obese Elementary School Children. $\mathrm{J}$ of Korean Academy of Nursing. 2007;37(6):902-913.

[11] Kelishadi R, Hashemipour M, Mohammadifard N, Alikhassy H, Adeli K. Short- and long-term relationships of serum ghrelin with changes in body composition and the metabolic syndrome in prepubescent obese children following two different weight loss programmes. Clin Endocrinol (Oxf). 2008;69(5):721-729.

[12] Reinehr T, Kleber M, Toschke AM. Lifestyle intervention in obese children is associated with a decrease of the metabolic syndrome prevalence. Atherosclerosis. 2009;207(1):174-180.

[13] Wickham EP, Stern M, Evans RK, Bryan DL, Moskowitz WB, Clore JN, Laver JH. Prevalence of the metabolic syndrome among obese adolescents enrolled in a multidisciplinary weight management program: clinical correlates and response to treatment. Metab Syndr Relat Disord. 2009;7(3):179-186.
[14] Hashemipour M, Kelishadi R, Shapouri J, Sarrafzadegan N, Amini M, Tavakoli N, Movahedian-Attar A, Mirmoghtadaee P, Poursafa P. Effect of zinc supplementation on insulin resistance and components of the metabolic syndrome in prepubertal obese children. Hormones (Athens). 2009;8(4):279-285.

[15] Pedrosa C, Oliveira BM, Albuquerque I, Simões-Pereira C, Vaz-de-Almeida MD, Correia F. Markers of metabolic syndrome in obese children before and after 1-year lifestyle intervention program. Eur J Nutr. 2011;50(6):391-400.

[16] Velázquez-López L, Santiago-Díaz G, Nava-Hernández J, Muñoz-Torres AV, Medina-Bravo P, Torres-Tamayo M. Mediterranean-style diet reduces metabolic syndrome components in obese children and adolescents with obesity. BMC Pediatr. 2014;175(14):1-10.

[17] Niklowitz P, Rothermel J, Lass N, Barth A, Reinehr T. Link between chemerin, central obesity, and parameters of the Metabolic Syndrome: findings from a longitudinal study in obese children participating in a lifestyle intervention. Int J Obes (Lond). 2018;42(10):1743-1752.

[18] Alberti KG, Eckel RH, Grundy SM, Zimmet PZ, Cleeman JI, Donato KA, et al. International Diabetes Federation Task Force on Epidemiology and Prevention; Hational Heart, Lung, and Blood Institute; American Heart Association; World Heart Federation; International Atherosclerosis Society; International Association for the Study of Obesity. Circulation. 2009 Oct 20;120(16):1640-5.

[19] Ladabaum U, Mannalithara A, Myer PA, Singh G. Obesity, abdominal obesity, physical activity, and caloric intake in US adults: 1988 to 2010. Am J Med. 2014;127(8):717-727.

[20] Méndez-Hernández P, Dosamantes-Carrasco LD, Siani C, et al. Mealtime habits and risk of developing the metabolic syndrome or insulin resistance among Mexican adults [published online ahead of print, 2016 Nov 15]. Br J Nutr. 2016;1-10.

[21] Rivas-Gómez B, Almeda-Valdés P, Tussié-Luna MT, AguilarSalinas CA. Dyslipidemia in Mexico, a call for action. Rev Invest Clin. 2018;70:211-216.

[22] Neri-Sánchez M, Martínez-Carrillo BE, Valdés-Ramos R, Soto-Piña AE, Vargas-Hernández JA, Benítez-Arciniega AD. Dietary patterns, central obesity and serum lipids concentration in Mexican adults. Nutr Hosp. 2019;36(1):109-117.

[23] Instituto Nacional de Estadística, Geografía e Informática / Instituto Nacional de Salud Pública / Secretaría de Salud. Encuesta Nacional de Salud y Nutrición 2018. Presentación de Resultados. Conociendo México. INEGIInforma. (s/f). 